\title{
Clinical Reviews
}

\section{Vinod Labhasetwar and Robert J. Levy}

\section{IMPLANTS FOR SITE-SPECIFIC DRUG DELIVERY}

Implantable drug delivery systems formulated from drug-polymer composites have been used recently in a variety of applications in which chronic administration of a pharmacological agent to a specific anatomic site is advantageous. These drug delivery systems are generally referred to as controlled release matrices. ${ }^{1}$ Their mechanism of drug release involves either passive drug diffusion, or biodegradation of the polymer matrix, or both. This review considers only those devices that are implanted at the drug target site, and not those that dispense drugs systemically. The advantages of sitespecific controlled release implants are that a substantially lower dose of the drug is required, since it is administered at an optimal location, and therefore the risks for systemic side effects are greatly reduced.

A controlled release implant, in widespread clinical use, is the dexamethasone-silicone rubber matrix configured for insertion in a cardiac pacing electrode tip. Although cardiac pacemakers have been utilized for the past 30 years, a persistent clinical problem has been the time-dependent progressive increase in the pacemaker electrode's electrical capture thresholds due to fibrosis. Dexamethasone is an antiinflammatory, antifibrotic synthetic steroid. The working hypothesis of the controlled release dexamethasone approach is that sustained release steroid administered directly to the endocardium contacting the electrode would limit acute inflammation and chronic inflammation, as well as the resultant scarring, that adversely affect electrical capture thresholds.

Several clinical studies have demonstrated the improved pacing threshold characteristics of a steroideluting electrode. A double-blinded study ${ }^{2}$ compared the same electrode configuration (Medtronic, Minneapolis, $\mathrm{MN}$ ) with and without steroid over a 2-year follow-up period. The results showed that from 2 weeks to 2 years the pulse duration thresholds for the steroid leads remained almost constant, while the nonsteroid cases demonstrated a significant rise in thresholds. A more recent double-blinded clinical study has confirmed these findings over a 6-month follow-up period. ${ }^{3}$ Thus, the steroid-eluting cardiac pacing lead represents an important example of an effective clinical use of a controlled release drug implant.

Requests for reprints should be sent to Robert J. Levy, M.D., Kresge II, Room 5014 POB 0576, University of Michigan, Ann Arbor, MI 48109.

Journal of Applied Biomaterials, Vol. 2, 211-212 (1991)

(C) 1991 John Wiley \& Sons, Inc.
Controlled release implants for brain tumor chemotherapy have also been recently shown to be clinically effective and advantageous. ${ }^{4}$ Glioblastoma multiforme is the commonest type of brain tumor, and its therapy via neurosurgery and drug administration is at present unsatisfactory. Very few patients with this tumor survive more than 3 months after the initial diagnosis if treated with conventional surgery and chemotherapy. The nitrosurea chemotherapeutic agent, carmustine (BCNU), has been the drug of choice for the systemic chemotherapy of this brain tumor following neurosurgical removal. However, this compound is very short acting and highly toxic. Severe systemic side effects greatly limit its usefulness.

Recurrent glioblastoma multiforme has been the focus of a clinical trial of controlled release implants containing $\mathrm{BCNU}$, that are placed in the tumor cavity after resection. These controlled release preparations consist of BCNU incorporated into a biodegradable high molecular weight polyanhydride. The polyanhydride formulation contains repeating subunits of sebacic acid and carboxyphenoxy-propane. The biocompatibility of this polyanhydride preparation has been well documented, ${ }^{5}$ and the initial clinical trials with BCNU-polyanhydride matrix implantation have been highly successful, with a mean survival time of 48 weeks following reoperation. In addition, because of the relatively low dose of BCNU used in the implants, the incidence of systemic side effects has also been reduced. At present, a multicenter double-blinded clinical trial is underway to further investigate the efficacy of this new approach.

The high molecular weight polyanhydrides offer a number of therapeutic options for further enhancements of this strategy. For example, the biodegradation of the high molecular weight polyanhydrides, and accompanying drug release, can take place over durations ranging from days to years, depending on the molecular weight and precise formulation parameters. Therefore, with the advent of new chemotherapeutic agents, and related anticancer strategies, it may be expected that the polyanhydrides will be appropriately utilized to enhance the treatment of a variety of localized solid tumors. In addition, the high molecular weight polyanhydrides have also been formulated as microspheres, ${ }^{6}$ which could be intravascularly injected into a tumor's vasculature to further enhance chemotherapy.

The surgical and medical therapy of osteomyelitis, as well as infected arthroplasties, has been fraught with difficulty due to problems optimizing antibiotic therapy. Antibiotic-impregnated bone cement has been used as a sustained release preparation for the treatment of established or potential bone infection. Most often, gentam- 
icin has been incorporated into bone cement matrices composed of polymethylmethacrylate derivatives. Thus, these base polymers serve a dual function as both bone cement and a controlled release matrix substrate for local therapy. An uncontrolled study of 27 patients with pedal infections treated with polymethylmethacrylate-gentamicin bone cement reported a $95 \%$ success rate based on freedom from further infection. ${ }^{7}$ However, the use of gentamicin-containing bone cement has also been associated with the emergence of antibiotic-resistant coagulase negative staphylococci. In a series of 91 patients with hip arthroplasties complicated by infection, ${ }^{8} 30$ patients developed gentamicin-resistant infections following treatment with antibiotic-impregnated bone cement. Thus, the ultimate usefulness of antibiotic-impregnated bone cement remains to be established by further clinical investigations.

Vascular grafts and cardiac valve prostheses with controlled release antibiotic formulations, have been utilized only to a limited extent clinically. A resorbable collagengentamicin controlled release matrix combined with a vascular graft composed of expanded polytetrafluoroethylene were reported to be effective for reducing the incidence of shunt infections in an uncontrolled series of 40 patients undergoing chronic hemodialysis. ${ }^{9}$ Furthermore, an antibiotic-controlled release system (gentamicin-polymethylmethacrylate) was also used in a perianular configuration to provide continuous antimicrobial administration to the immediate vicinity of a mechanical cardiac valve prosthesis implant in a case of aortic root endocarditis. ${ }^{10}$ Although no information was provided in this case report concerning the duration of drug release, the patient receiving the implant was improved one year postoperation.

In summary, site-specific drug implants represent a new and innovative therapeutic approach, which will very likely become of general importance for treating a number of localized disease processes. However, there is an obvious need for controlled clinical trials to evaluate efficacy. Furthermore, the controlled release systems described in this review are based on conventional pharmacologic agents. Thus, it may be expected that in the future site-specific controlled release will involve custom-synthesized agents, which may be targeted more precisely to the tissue or organ of interest. In addition, controlled release implants as drug carriers for site-specific gene therapy may also be expected to be developed.

\section{REFERENCES}

1. Langer, R. New methods of drug delivery. Science 249:1527-1533; 1990.

2. Mond, $\mathbf{H}$. et al. The porous titanium steroid eluting electrode: a double blind study assessing the stimulation threshold effects of steroid. PACE 11:214-219; 1988.

3. Wish, M. et al. Steroid-tipped leads versus porous platinum permanent pacemaker leads: a controlled study. PACE 13:1887-1890; 1990.

4. Brem, H. et al. Interstitial chemotherapy with drug polymer implants for the treatment of recurrent gliomas. J. Neurosurg. 74:441-446; 1991.

5. Tamargo, R. J. et al. Brain biocompatibility of a biodegradable, controlled-release polymer in rats. J. Biomed. Mater. Res. 23:253-266; 1989.

6. Bindschaedler, C. et al. Polyanhydride microsphere formulation by solvent extraction. J. Pharm. Sci. 77:696-698; 1988.

7. Stabile, D. E.; Jacobs, A. M. Local antibiotic treatment of soft tissue and bone infections of the foot. J. Am. Podiatr. Med. Assoc. 80:345-353; 1990.

8. Hope, P. G. et al. Deep infection of cemented total hip arthroplasties caused by coagulase-negative staphylococci. J. Bone Jt. Surg. 71:851-855; 1989.

9. Horch, R.; Kronung, G. Prevention of infection in teflon prostheses for dialysis access. Experiences with a resorbable combined collagen-antibiotic system. Vasa 18:3034; 1989.

10. Faidutti, B. et al. Implantation of antibiotic-releasing carriers for treatment of recurrent prosthetic endocarditis. J. Thorac. Cardiovasc. Surg. 92:159-161; 1986. 